\title{
LOS DERECHOS HUMANOS Y LOS DEPORTES JUVENILES ${ }^{1}$
}

\author{
Vitaly Melnikov \\ Vice-President of the Central Olympic Academy of Russia, \\ PhD, Associate Professor \\ melnikov_vitalik@yandex.ru
}

Fecha de recepción: septiembre 2020

Fecha de aceptación: noviembre 2020

http://doi.org/10.15366/citius2020.13.2.002

\section{Resumen:}

El presente trabajo pretende, a partir de la información recogida en la documentación de diversas estructuras internacionales, mostrar la importancia del deporte en el colectivo juvenil como un Derecho Humano fundamental en el panorama internacional. Asimismo, se recoge el papel fundamental que han tenido las estructuras olímpicas en el desarrollo del deporte para este colectivo, enfatizando la importancia de valores como la amistad, las relaciones culturales internacionales, el respeto y la tolerancia, más allá de los resultados deportivos; todo ello con el objetivo de formar a la juventud mundial y conseguir la paz universal. El presente trabajo presenta así una revisión de las diversas ediciones deportivas nacionales e internacionales y su evolución, mostrando un incremento positivo en todos los casos. Por último, se expone de manera detallada uno de los eventos deportivos que se creó y evolucionó con la misma finalidad olímpica citada: El Foro Internacional de Jóvenes Olímpicos de Rusia, que se llevó a cabo con el fin de proteger a los atletas de los actuales fenómenos negativos del deporte y en particular del dopaje.

Palabras clave: Derechos Humanos, Deporte y Juventud, Olimpismo, mega eventos deportivos

\section{Title: HUMAN RIGHTS AND YOUTH SPORTS}

\begin{abstract}
:
This work aims, from the information collected in the documentation of various international structures, to show the importance of sport in the youth group as a fundamental human right in the international scene. Likewise, the fundamental role that Olympic structures have had in the development of sport for this group is collected, emphasizing the importance of values such as friendship, international cultural relations, respect and tolerance beyond sport results, all of this with the aim of educating the world's youth and achieving universal peace. The present work thus presents a review of the various national and international sports editions and their evolution, showing a positive increase in all cases. Finally, one of the sports events which was created and developed with the same Olympic purpose mentioned, The International Forum of Olympic Youth in Russia, is developed in detail with the aim of protecting the athletes from the current negative phenomena in sport, particularly from doping.
\end{abstract}

Keywords: Human Rights, Sport and Youth, Olympic Movement, mega sport events

\section{El deporte como un derecho humano fundamental en la juventud}

Los Derechos Humanos ocupan un papel fundamental en los documentos de las Naciones Unidas, del Consejo Europeo y de otras organizaciones internacionales. En particular, en la resolución titulada «Promoción de los derechos humanos mediante el deporte y los ideales del Movimiento Olímpico» (United Nations Human Right Council, 2013) en ella se enfatiza sobre la importancia del deporte como un vehículo para la promoción de los Derechos Humanos. Se constata así, que el potencial unificador del deporte y la armonía de los valores e ideales del Movimiento Olímpico pueden y deben utilizarse ampliamente para promover las normas de derechos humanos universalmente reconocidas. Esto se adapta plenamente al desarrollo del deporte entre los jóvenes.

\footnotetext{
${ }^{1}$ El texto del presente artículo es la versión en español del trabajo presentado en inglés por su autor en la $16^{\text {th }}$ International Session for Directors of NOAs celebrada desde la sede de la Academia Olímpica Internacional en Olimpia (Grecia) y que fue expuesta el día 11 de septiembre del 2020. Ha sido traducido por la Dra. Ma Teresa Calle Molina.
} 
En la citada resolución se incita a los Estados a que cooperen con los Comités Olímpicos y Paralímpicos internacionales en planteamientos prioritarios como la superación de la discriminación, el logro de la igualdad y el fortalecimiento de la cooperación y el respeto mutuo en el contexto de la protección de los Derechos Humanos.

La aprobación por consenso de la resolución «Promoción de los derechos humanos mediante el deporte y los ideales del Movimiento Olímpico», combinada con un número sin precedentes de coautores, hasta 130, es una prueba más de que la interacción constructiva y despolitizada entre los Estados sobre cuestiones fundamentales de Derechos Humanos está dando resultados positivos. Por tanto, podemos afirmar, que el derecho de los jóvenes a practicar deportes es una de las prioridades más importantes entre los derechos humanos fundamentales.

Los jóvenes de hoy en día, provenientes de diferentes países, practican una variedad de deportes que satisfacen diferentes gustos y caracteres y, por lo tanto, en algún momento, el deporte se ve estrechamente relacionado con la identidad y la cultura de una persona. Si se examinan a fondo los valores y objetivos básicos de los deportes y los juegos —incluidos los juegos infantiles - es evidente que todos los deportes, ya sea el fútbol, el atletismo o la natación, se convierten en un medio de enseñanza de aptitudes esenciales para la vida; y por eso, los deportes se consideran una parte importante de los programas de educación de los jóvenes. Es por ello por lo que, es necesario crear condiciones en las que los jóvenes disfruten plenamente de los derechos humanos básicos y también —en igualdad de condiciones con los adultos - puedan tener garantías sociales, incluida la oportunidad de practicar deportes y cultivar un estilo de vida saludable.

\section{El papel del olimpismo en el desarrollo del deporte en la población joven.}

El Movimiento Olímpico moderno tiene una gran importancia social y contribuye al establecimiento de vínculos entre las organizaciones deportivas de diversos países. En este movimiento social, política e ideológicamente complejo, son fundamentales principios progresistas como la inadmisibilidad de la discriminación política, racial y religiosa en el deporte, el reconocimiento de la soberanía e igualdad de las organizaciones deportivas nacionales, la no injerencia en la vida privada, los fundamentos democráticos de la organización del movimiento y la participación en los impulsos por lograr la paz universal.

La adhesión inquebrantable a estos principios es la principal condición para asegurar la unidad del Movimiento Olímpico y el progreso de los Juegos Olímpicos (JJOO). Es también una parte importante de la educación olímpica, que el Comité Olímpico Internacional (COI), Los Comités Olímpicos Nacionales (CONs) y la Academia Olímpica Internacional (AOI) y las Academias Olímpicas Nacionales (AONs) llevan a cabo con éxito en los diversos países del mundo.

El Movimiento Olímpico une a millones de jóvenes, sin importar sus opiniones políticas y religiosas o su raza. Los múltiples esfuerzos por la aplicación de los principios olímpicos progresistas expresan la esencia democrática general del Movimiento Olímpico. La idea de los deportes juveniles pacíficos, la cooperación internacional y el logro de la comprensión mutua y la amistad entre los pueblos encuentran su expresión concreta en los Juegos Olímpicos.

De acuerdo con el párrafo 2.4 del artículo 31 de la Carta Olímpica, los Comités Olímpicos Nacionales promueven la creación de organizaciones especializadas dedicadas a la educación olímpica. En particular, éstos están comprometidos con el establecimiento y funcionamiento de Academias Olímpicas Nacionales, museos olímpicos y la creación de programas culturales relacionados con el Movimiento Olímpico. Así, estas instituciones, en sus respectivos países, deben ayudar a capacitar al personal deportivo y promover los Principios Fundamentales del Olimpismo (International Olympic Committee, 2020).

Por otra parte, los principios especiales del derecho olímpico reflejan la esencia y las leyes objetivas del desarrollo de las relaciones olímpicas internacionales. 
El Movimiento Olímpico se esfuerza por modelar su futuro de acuerdo con las exigencias de la época. En la sesión del COI celebrada en Mónaco en 2014 se aprobó la «Agenda Olímpica 2020», que contiene 40 recomendaciones sobre las tendencias de desarrollo de los Juegos Olímpicos hasta el año 2020. En este documento se puede apreciar cómo varias recomendaciones se refieren al desarrollo de los deportes juveniles. Así, el Movimiento Olímpico no sólo conservará su poder e influencia en los deportes, sino que lo fortalecerá significativamente integrándolos en los deportes y disciplinas profesionales actualmente independientes (International Olympic Committee, 2014)

Al mismo tiempo, los CONs deben llevar a cabo una labor de propaganda que promueva los valores e ideas del olimpismo, fomente el desarrollo de los deportes de alto rendimiento y los deportes de masas, participe en la capacitación y formación de las personas que se integran profesionalmente en el ámbito deportivo, impida la discriminación y la violencia en el deporte, y aplique el código mundial antidopaje.

\section{Mega eventos deportivos destinados a la participación de población joven}

\section{1.- Una revisión internacional}

Actualmente, el deporte juvenil está atrayendo cada vez más atención. En las últimas décadas, han aparecido muchas formas de competiciones internacionales importantes entre los jóvenes, siendo el principal los Juegos Olímpicos de la Juventud. Un rasgo importante de todas estas competiciones es el hecho de que no se centran en el logro de resultados deportivos sobresalientes; sino en la formación de hombres y mujeres jóvenes y en el beneficio de la amistad, el respeto y la comprensión entre personas de diferentes nacionalidades. La participación de los jóvenes atletas en competiciones de esta índole es un acontecimiento significativo en su carrera deportiva y, para algunos de ellos, es el primer paso hacia las victorias deportivas.

Los principales factores que determinan el impacto en el proceso de participación de los jóvenes en el Movimiento Olímpico y deportivo son:

— El apoyo de los gobiernos de muchos países al movimiento deportivo juvenil.

- Subvenciones para programas de desarrollo de los deportes juveniles y mejora del sistema de deportes de alto rendimiento.

- Elaboración y aplicación de programas para mejorar la aptitud física de los jóvenes a nivel nacional.

— Inclusión en la “Agenda 2020”, por parte del Comité Olímpico Internacional, de una serie de normas y reglamentos sobre la participación de los jóvenes en el Movimiento Olímpico que se sustentan en la creación de condiciones innovadoras para la organización y realización de competiciones internacionales, regionales y nacionales.

La primera competición específica entre los jóvenes dentro de la estructura olímpica se organizó como los Días Olímpicos de la Juventud Europea. En 1990, se decidió celebrar este acontecimiento una vez cada dos años, y pasó a llamarse Festival Olímpico de la Juventud Europea (FOJE), El creador de estas competiciones fue Jacques Rogge, quien dirigía la Asociación de Comités Olímpicos Europeos de la época. Estos festivales de verano se han celebrado desde 1991, y los de invierno desde 1993.

Asimismo, los Juegos Mundiales de la Juventud (World Youth Games), celebrados en Moscú en 1998, marcaron una nueva forma de organización de macro competiciones internacionales para niños y niñas. En la actualidad, han supuesto la mayor competición organizada de este tipo, más de 7000 atletas de 131 países de los cinco continentes participaron en ella. El objetivo principal de estos Juegos fue involucrar a los jóvenes deportistas en el Movimiento Olímpico, prepararlos para las difíciles condiciones psicológicas de las competiciones internacionales y seleccionar jóvenes talentos para participar en futuros Juegos Olímpicos. La ceremonia de apertura incluyó todos los protocolos que se venían utilizando de 
manera tradicional en unos Juegos Olímpicos, incluyendo el desfile de equipos en la ceremonia inaugural y el encendido de la llama olímpica.

Por otra parte, la competición deportiva internacional de los Juegos Niños de Asia surgió, por una parte, debido a la necesidad objetiva de ampliar el sistema de competiciones deportivas para los niños y jóvenes en la región de Asia-Pacífico y, por otra parte, como resultado de la necesidad de hacer participar a la generación más joven en la cultura física y los deportes. Las competiciones internacionales de nivel regional, como el Festival Olímpico de la Juventud Europea, el Festival Olímpico Juvenil de Australia y otras competiciones y torneos de nivel regional en el sistema de deporte juvenil internacional se han convertido en requisitos previos para este tipo de competición. Una característica distintiva de los Juegos Niños de Asia es que pone en práctica el potencial positivo de las actividades deportivas, teniendo en cuenta la elaboración y ejecución de diversos proyectos y programas basados en las tradiciones nacionales y en los principios olímpicos de orientación humanitaria.

El Festival Olímpico Juvenil de Australia fue iniciado por el Comité Olímpico Nacional de Australia después de los XXVII Juegos Olímpicos de Sídney en 2000. Éstos se celebran bajo el patrocinio del COI, financiados en su totalidad por el Comité Olímpico Nacional de Australia.

Los Juegos Asiáticos de la Juventud se celebran cada cuatro años, además de los Juegos Asiáticos. Los primeros Juegos Asiáticos se celebraron en 2009 en Singapur, entre el 29 de junio y el 7 de julio. Los Juegos Asiáticos de la Juventud se introdujeron por primera vez en 2007, como parte de los Juegos Olimpicos de la Juventud, con la finalidad de clasificar a sus deportistas provenientes de los países asiáticos en los Juegos Olímpicos de la Juventud.

Así, podemos concretar que los principales objetivos de los Juegos Asiáticos de la Juventud son:

- Reunir a jóvenes de toda Asia para la competición y el entrenamiento deportivo.

- Brindar la oportunidad de unirse a los valores olímpicos con el fin de lograr la excelencia, la amistad y el respeto.

- Crear una plataforma para la amistad y el intercambio de valores culturales entre los jóvenes participantes.

Además de los deportes, los Juegos Asiáticos de la Juventud incluyen un programa educativo de gran relevancia, el cual se centra en la promoción y mantenimiento de un equilibrio adecuado entre las áreas deportiva, cultural y educativa que se integran en los Juegos.

Por otra parte, los Juegos Olímpicos de la Juventud son una compleja competición deportiva internacional en el sistema de deportes infantiles y juveniles. Las características socio-pedagógicas de este tipo de competición son algunas como: la concentración de los mejores atletas jóvenes para participar en competiciones multideportivas de alto nivel en un festival deportivo; un formato que también incluye eventos educativos y culturales; la promoción del intercambio cultural y de los valores olímpicos, incluyendo la no discriminación, el juego limpio y la búsqueda de la excelencia; la interacción con los jóvenes a través de las plataformas digitales del Movimiento Olímpico (incluido el canal olímpico y la página web oficial del COI) y la utilización del legado de las competiciones deportivas juveniles internacionales (como son el Festival Olímpico de la Juventud Europea desde 1991, el Festival Olímpico Juvenil de Australia desde 2001, los Juegos Asiáticos de la Juventud desde 2009 y los Juegos Olímpicos de la Juventud de 1998).

El análisis de los principales indicadores de los Juegos Olímpicos de la Juventud muestra una evolución positiva en el número de atletas, deportes y en el número de países participantes. Así, se puede observar que, durante los Juegos Olímpicos de la Juventud de verano, el número de deportes aumentó de 26 a 31, el número de participantes aumentó de 3524 a 4012, y el número de países participantes aumentó de 204 a 206. Asimismo, en las ediciones de invierno el número de deportes ha aumentado de 7 a 8 , el 
número de participantes de 1020 a 1880, y el número de países participantes se ha incrementado de 69 a 79.

El sistema de competiciones deportivas para niños y jóvenes en un país determinado incluye una gran cantidad de eventos deportivos y de cultura física destinados a desarrollar el sistema de educación física y a aumentar el número de personas que participan sistemáticamente en la cultura física y los deportes. Por lo que la participación de la generación más joven en la educación física y los deportes basados en los valores olímpicos es una dirección prioritaria para el desarrollo de la educación física y los deportes en Rusia.

\subsection{El Foro Internacional de Jóvenes Olímpicos}

El Foro Internacional de Jóvenes Olímpicos se ha convertido en una de las formas educativas de atraer a los jóvenes a los conocimientos olímpicos. Éste supone un evento educativo organizado por el Comité Olímpico Ruso en cooperación con el Ministerio de Deportes de Rusia, el Organismo Ruso de Lucha contra el Dopaje y el Departamento de Deportes y Turismo de Moscú con el apoyo del COI (RUSADA, 2020). Los principales objetivos del Foro son crear y difundir una cultura antidopaje y un rechazo consciente de los fenómenos negativos en el deporte entre los jóvenes atletas.

La primera edición del Foro de Jóvenes Olímpicos se celebró en Moscú el 1 de junio de 2018. Asistieron más de 300 jóvenes atletas rusos. Durante su celebración, los jóvenes atletas tuvieron formación de los representantes del Ministerio de Deportes de Rusia, del Organismo Ruso de Lucha contra el Dopaje y de la Agencia Mundial Antidopaje sobre las principales normas antidopaje y la responsabilidad de su violación, así como sobre los detalles del procedimiento de control del dopaje en los mega eventos deportivos. El Foro contó con la participación de los mejores atletas rusos, que habían sido campeones olímpicos en diversas ediciones de los Juegos Olímpicos de invierno y verano.

La segunda edición del citado Foro se celebró el 1 de junio de 2019, coincidiendo con en el Día Mundial de la Infancia. En esta ocasión este evento tuvo categoría de internacional. Asistieron más de 100 atletas de 16 países: Rusia, Hungría, Eslovaquia, Serbia, Eslovenia, Polonia, Bulgaria, Georgia, Armenia, Turkmenistán, Kazajstán, Azerbaiyán, Uzbekistán, Bielorrusia, Kirguistán y Tayikistán.

El objetivo principal del Foro es proteger a los jóvenes atletas de los actuales fenómenos negativos que existen en el deporte, el principal de los cuales es el dopaje. Las sedes y los programas de entrenamiento fueron diseñados para un público joven, ya que esta categoría de edad particular de los atletas es una prioridad para el Comité Olímpico Ruso, que cuenta con el apoyo activo del COI, la Agencia Internacional Antidopaje y Organismo Ruso de Lucha contra el Dopaje.

La comunicación se realizó en dos idiomas: ruso e inglés. El Foro Internacional de Jóvenes Olímpicos se ha convertido en un evento anual. Respecto a la organización y celebración de mega eventos deportivos en el ámbito de los deportes juveniles, se denota un aumento del prestigio del país anfitrión y de los países participantes por parte de la comunidad deportiva mundial, y esto contribuye a atraer inversiones extranjeras, mejorando las relaciones internacionales, en particular mediante una intensa cooperación en la preparación y organización de los Juegos. Todo ello, a su vez, repercute positivamente en el desarrollo de diversos factores, como la economía, las finanzas, el desarrollo de los deportes de masas, el movimiento de voluntarios y el fortalecimiento de las relaciones diplomáticas entre los diversos países.

Por todos estos aspectos, podemos concluir que es la diplomacia deportiva la que desempeña un papel importante en este proceso, que contribuye a mejorar la comprensión mutua y a desarrollar una amplia cooperación entre los países de todo el mundo. 


\section{Referencias bibliográficas}

- International Olympic Committee (2014) Olympic Agenda 2020 (20+20 Recomendations). Recuperado de https://stillmed.olympic.org/Documents/Olympic_Agenda_2020/Olympic_Agenda_2020-2020_Recommendations-ENG.pdf

- International Olympic Committee (2020). International Olympic Charter 2020. Lausana, Suiza. Recuperada de https://stillmed.olympic.org/media/Document\%20Library/OlympicOrg/General/ESOlympic-Charter.pdf

- Rusada (2020). RUSADA: Forum for Young Olympians. Recuperado de https://rusada.ru/en/events/conferences/forum-yunykh-olimpiytsev/

- United Nations Human Right Council (2013). Sport and Olympic Ideal. Recuperado de https://www.ohchr.org/EN/HRBodies/HRC/AdvisoryCommittee/Pages/HumanRightsThroughSport.a $\underline{\operatorname{spx}}$ 